\title{
CRACK GROWTH IN CONCRETE UNDER UNIAXIAL AND CONFINED CONDITIONS
}

\author{
KAMRAN M. NEMATI ${ }^{*}$ AND JOHN KEMENY ${ }^{\dagger}$ \\ *University of Washington \\ Seattle, WA USA \\ e-mail: nemati@uw.edu \\ ${ }^{\dagger}$ University of Arizona \\ Tucson, AZ USA \\ e-mail: kemeny@email.arizona.edu
}

Key words: Concrete, microcracks, Crack growth, Crack density, Uniaxial compression, Confining stress

\begin{abstract}
This paper compares the results of the experimental compressive stress-induced microcracks and numerically simulated compressive microcracks in concrete under uniaxial and triaxial loading condition. An alloy with a low melting point was used to preserve the microcracks in concrete as they exist under load. Scanning electron microscope was used to capture images from the cross sections of concrete specimens. These images were then used to determine length, orientation, and density of the compressive stressed-induced microcracks in concrete specimens. A crack growth simulation model was used to generate and propagate microcracks for uniaxial and triaxial loading conditions. The crack growth simulation model revealed results similar to that obtained from the experiments.
\end{abstract}

\section{INTRODUCTION}

The preservation of stress-induced microcracks and the identification of cracks induced by loading is important for understanding the mechanisms for the formation, propagation and interaction of stress-induced microcracks in concrete.

Since the 1960s, researchers have suggested and assumed the existence of different kinds of defects called microcracks that occurs in concrete. Since the early 1980s, these microcracks have been verified and, to some extent, defined by microscopic study. Interfacial transition zone (ITZ) cracks may occur at mortar-aggregate interfaces, while mortar and aggregate cracks occur in the mortar and aggregate, respectively ${ }^{1}$

Although the general relationships between stress level and crack development are reasonably well understood, the origin and development of cracks in three-phase concrete composite systems is still uncertain. In an effort to explore these issues, linear elastic fracture mechanics (LEFM) have been extended and applied to concrete; however, these studies have not been very successful, perhaps, in part, because the observations they made into the way small cracks are formed and then propagate in these materials was very limited. Only as early as 1960's have such cracks been carefully observed, measured, and characterized in interior portions of the system. 
Several methods have been used to study the microcracking of concrete. Some of those methods are: Sonic testing, Acoustic emission, Mercury intrusion porosimetry, Hydrophilic tracer liquid technique, Microscope technique with dye, and X-ray technique which made it possible to observe the interior cracks within the concrete rather than on cracks on an exterior surface. Almost all the techniques employed were ineffective and did not represent the actual geometry and state of microcracks as they exist under a load. Scanning Electron Microscopy (SEM) has made possible the detailed observation of microcracks in concrete. Yadev ${ }^{2}$ used a metal in liquid phase called "Wood's metal" that has a melting point below the boiling point of water to study pore fluid porosimetry and to measure contact areas and voids between the surfaces of natural fractures. To preserve the microstructure in specimens under load, Zheng ${ }^{3}$ used Wood's metal to fill voids and microcracks in rock during loading and solidified it in place before unloading. Employing the method introduced by Zheng ${ }^{3}$, in this study microcracks induced in concrete by applied stresses were preserved and identified in order to understand the mechanisms by which stress-induced microcracks generate, propagate, and interact.

\section{EXPERIMENTAL METHOD}

The test equipment and experimental method has been described in detail elsewhere (Nemati et al. ${ }^{4}$ ). The experiments involved three procedures: a) concrete casting and preparation, b) crack induction, and c) molten metal injection and solidification. The second and third procedures were carried out simultaneously.

Concrete cylinders of 8 -inches long by 4inches in diameter, were tested at the Structural Engineering Laboratory of the University of California at Berkeley. In order to preserve the microstructure and microcracks in concrete specimens under load, the voids and microcracks were filled with liquid "wood's metal" during loading, and the alloy was solidified in place before unloading. Woods metal, consisting of $42.5 \% \mathrm{Bi}, 37.7 \% \mathrm{~Pb}$,
$11.3 \% \mathrm{Sn}$, and $8.5 \% \mathrm{Cd}$, has a melting point range from $160^{\circ} \mathrm{F}$ to $190^{\circ} \mathrm{F}$ and is solid at room temperature. Cylindrical concrete specimens were submerged in the molten metal alloy during the test and the alloy was driven into voids and fractures by pore pressure provided by nitrogen gas. The alloy could be solidified at any stage of the experiment to preserve the geometry of microcracks as they existed under load and to distinguish them from those generated later. With a surface tension of 400 $\mathrm{mN} / \mathrm{m}$, the alloy could penetrate into flat cracks with apertures as fine as 0.08 microns under a pore pressure of 1500 psi. Such a technique not only allows one to observe the microcracks as they exist under load (Figure 1), but also facilitates observing the microcracks in three dimensions $^{5}$ (Figure 2).

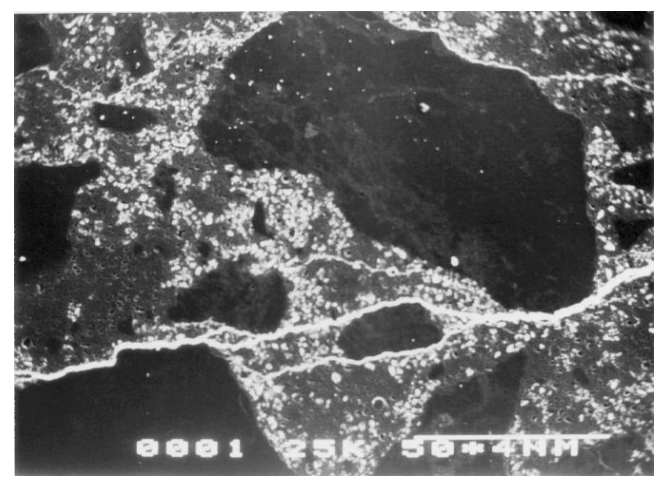

Figure 1 Micrograph of a stress-induced concrete specimen

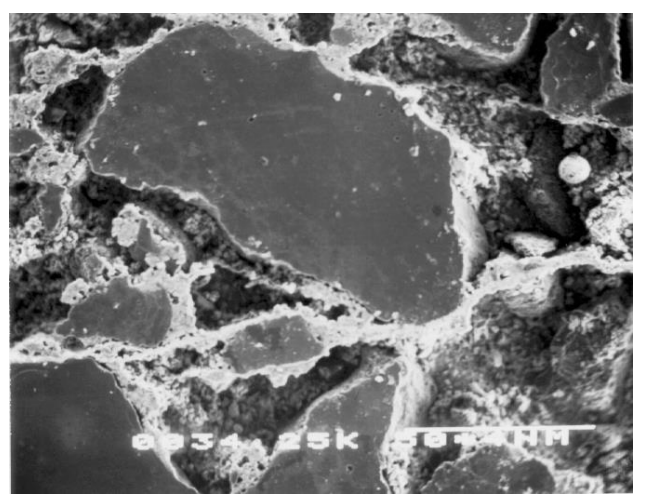

Figure 2 Micrograph of the same image as Figure 1 with the cement paste removed to reveal cracking in three dimensions 


\section{IMAGE ANALYSIS}

After the metal was solidified, each of the cylinders was sectioned into eight specimens. The specimens were observed using JEOL JSM-35CF Scanning Electron Microscopy (SEM). The SEM images were then analyzed using an image analyzer. In order to identify Wood's metal, which is representative of pores and fractures in concrete, the image analyzer can make a histogram for all of the different phases in the image based on their gray levels, with zero representing the darkest phase and 255 representing the brightest phase. From this histogram, and by means of trial and error method, two threshold levels can be established to encompass the brightest phase in the image, namely Wood's metal ${ }^{1}$.

\section{CRACK GROWTH}

The energy release rate is usually defined as the energy released from the body per unit crack advance. A more precise definition ${ }^{6}$ involves the work input into the crack tip. Irwin ${ }^{7}$ defined an energy release rate, $G$, which is a measure of the energy available for an increment of crack extension. Considering the plate, shown in Figure 3, with a thickness $B$, and containing a crack with a length of $2 a^{8}$.

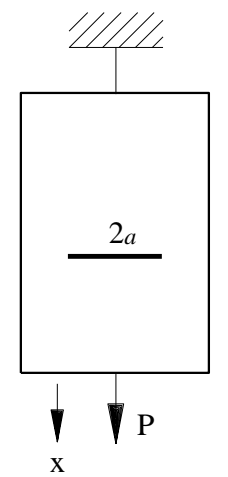

(a)

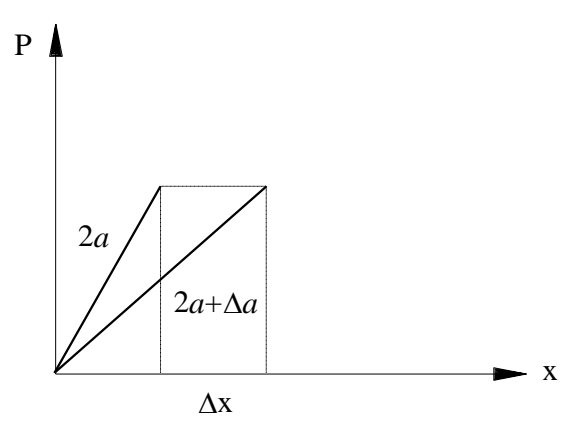

(b)
Figure 3 (a) Plate with crack $2 a$; (b) Loaddisplacement diagram

If the plate is subjected to a constant load $P$, the energy released can be expressed by crack growth $\Delta a$ as:

$$
G B \Delta a=P \Delta x-\Delta U_{e}
$$

where $\Delta U_{e}$ is the change in elastic energy due to crack growth $\Delta a$. In the limit:
$G B=P \frac{d x}{d a}-\frac{d U_{e}}{d a}$

the strain energy $U_{e}$ in terms of compliance is given by:

$\Delta U_{e}=\frac{c P^{2}}{2}$

and Equation 2 becomes:

$G=\frac{P^{2}}{2 B} \frac{d c}{d a}$

Where: $G=$ energy release rate

$P=$ applied load

$B=$ plate thickness

$c=$ compliance $($ displacement/load $)$

$a=$ half-crack length

Irwin $^{9}$ defined the quantity $G_{c}$ as the work required to produce a unit increase in crack area, referred to as critical energy release rate. $G_{c}$ is a material property and is determined experimentally. In order to determine whether or not a crack will propagate, the value of energy release per unit increase crack area, $G$, is computed. If the energy release rate is lower than the critical energy release rate $\left(G<G_{c}\right)$, the crack is stable. Conversely, if $G>G_{c}$, the crack will propagate. A condition in which the energy release rate equals the critical energy release rate $\left(G=G_{c}\right)$ is known as metastable equilibrium.

The stress intensity factor, $K_{I}$, is defined as:

$$
K_{I}=\sigma \sqrt{a} f(g)
$$

Where: $\quad K_{I}=$ stress intensity factor for mode I ( stress $\sqrt{\text { length }})$

$\sigma=$ applied stress

$f(g)=$ a function depending on the specimen and crack geometry

These two parameters, the energy release rate and the stress intensity factor, describe the 
behavior of cracks. The former quantifies the net change in potential energy that accompanies an increment of crack extension; the latter characterizes the stresses, strains, and displacements close to the crack tip. The energy release rate describes global behavior, whereas the stress intensity factor is a local parameter. For linear elastic materials, $K_{I}$ and $G_{c}$ are uniquely related. Considering only mode I and plane stress condition, for linear elastic behavior:

$G=\frac{K_{I}^{2}}{E}$

The critical stress intensity factor, $K_{c}$, commonly known as fracture toughness, is assumed to be a material property.

The $G$-criterion, usually called the maximum energy release rate, states that:

a) Crack initiation takes place at the crack tip and in a direction with respect to the original crack plane.

b) Crack extension takes place in the direction along which the strain energy release rate is maximum.

c) Crack initiation occurs when the maximum strain energy release rate in a direction reaches a critical value.

\section{CRACK GROWTH SIMULATION MODEL}

$\mathrm{Du}^{10}$ has developed a micromechanical model by which, given the initial crack conditions in an unloaded specimen, the final cracking state can be predicted for different loading conditions. The experimental results will be compared to the theoretical results using Du's crack-growth model. Du's model employs three commonly used fracture criteria: the maximum stress criterion; the maximum energy release rate criterion; and the minimum strain energy density criterion. The criterion most commonly used for concrete is the maximum energy release rate criterion. The maximum energy release rate criterion states that:
- Crack initiation takes place at the crack tip and in a direction with respect to the original crack plane.

- Crack extension takes place in the direction along which the strain energy release rate is maximum.

- Crack initiation occurs when the maximum strain energy release rate in a direction reaches a critical value.

The $\mathrm{Du}$ model uses the displacement discontinuity method which allows the fracture mechanics parameters to be readily computed for a given crack problem. By assuming a virtual crack increment, the crack initiation direction can be determined by one of the three fracture initiation criteria discussed above. The crack propagation path can be determined by assuming several crack increments. After each crack increment, the computer program recalculates the fracture parameters and the corresponding initial angle of crack propagation is determined. Figure 4 is the flow chart of the Du model computer program.

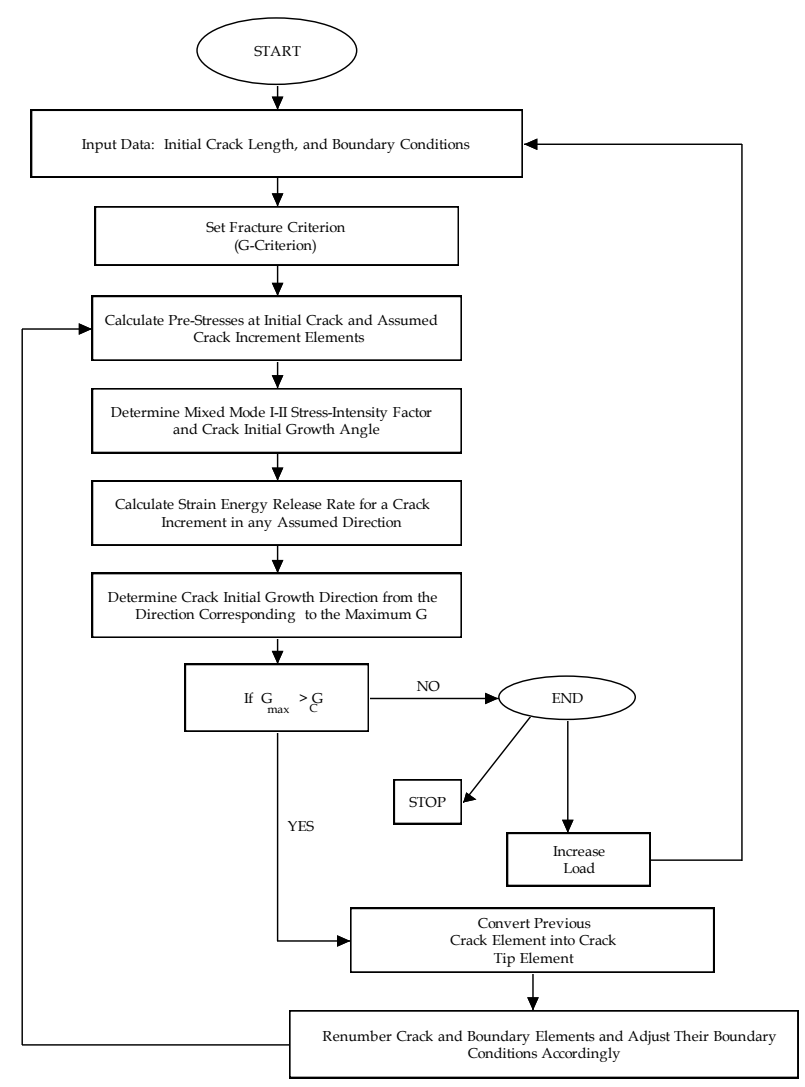

Figure 4 Computer flow chart for the $\mathrm{Du}$ model calculations 
The purpose of the $\mathrm{Du}$ model is to numerically simulate the behavior of stochastic distributions of heterogeneous microcracks. This behavior is difficult to model using analytical models. Du's micromechanical model is based on the idea that frictional sliding along pre-existing cracks results in the formation of tension cracks at their tips. The basis for the model is the displacement discontinuity model of Crouch and Starfield ${ }^{11}$. This model is based on the analytical solution to the problem of a constant discontinuity in displacement over a finite line segment in the $\mathrm{x}, \mathrm{y}$ plane on an infinite elastic solid. A displacement discontinuity can be visualized physically as a line crack whose opposing surface have been displaced relative to one another. In the case under consideration here, surfaces are displaced relatively by a constant amount along the entire crack. However, in general, one could consider an arbitrary distribution of relative displacement.

This method is based on the notion that one can make a discrete approximation of a continuous distribution of displacement discontinuity along a crack. That is, a crack can be divided into a series of $N$ elements (boundary elements) and the displacement discontinuity assumed to be constant over each one. On the basis of the analytical solution for a single, constant element displacement discontinuity, we derive a numerical solution to the problem by summing up the effects of all $N$ elements. When the distribution of displacement discontinuity along the crack is not known, the distribution of traction applied to the crack surfaces must be known in order to define the problem properly. The values of the element displacement discontinuities necessary to produce these tractions, are then sought, element by element along the $\operatorname{crack}^{11}$. The microcracks yielded by the no-load experiment, were used as the initial input microcracks in the Du model.

Table 1 is the tabulation of the microcracks used in the model.
Table 1 The microcrack lengths used in the Du model

\begin{tabular}{|c|c|}
\hline \multicolumn{2}{|c|}{ NORMAL-STRENGTH CONCRETE } \\
\hline Crack Length (mm) & Frequency \\
\hline \hline 0.4 & 26 \\
\hline 0.5 & 18 \\
\hline 0.6 & 4 \\
\hline 0.7 & 4 \\
\hline 0.8 & 3 \\
\hline 0.9 & 1 \\
\hline 1.0 & 1 \\
\hline 1.3 & 1 \\
\hline 1.5 & 1 \\
\hline Total & 59 \\
\hline
\end{tabular}

Crack lengths smaller than $0.4 \mathrm{~mm}$ are ignored and, because crack apertures are negligibly small, line cracks were assumed. The Du model uses the Monte Carlo technique to generate a distribution of line cracks. Monte Carlo simulation is used for problems involving random variables with known (or assumed) probability distribution.

Because of the assumption of a uniformly random distribution of initial crack locations and orientation, an algorithm has been developed to generate a similar distribution. For each crack generated, three random numbers are initially picked to determine the crack position $(\mathrm{x}, \mathrm{y})$, orientation, and according to Table 1, a non-random crack generation is implemented to generate a distribution of crack length $\boldsymbol{L}^{12}$. The crack orientations are between 0 and 90 degrees. A tree-cutting algorithm is used to eliminate cracks that intersect. Figure 5 is an illustration of the cracks generated in a concrete specimen with rectangular boundaries. 


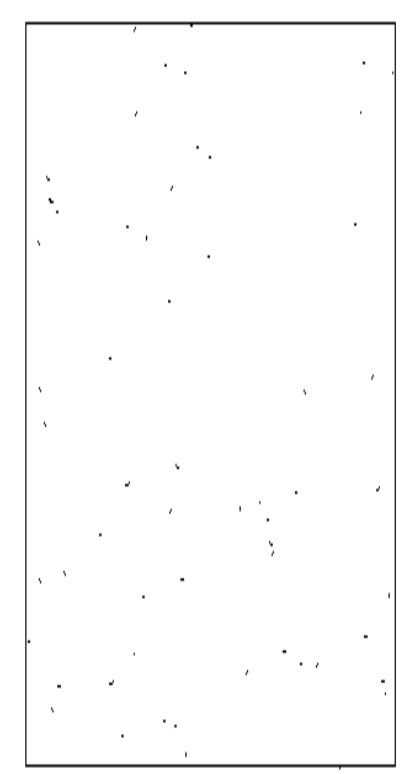

Figure 5 The random cracks generated in concrete specimen

\section{Parameters Used in the Crack Growth Model}

The CEB-FIP model code 2010 recommends the use of the following expression for the energy release rate:

$$
G_{f}=\alpha_{f}\left(f_{c m} / f_{\text {cmo }}\right)^{0.7}
$$

where $\alpha_{f}$ is a coefficient which depends on the maximum aggregate size $d_{\max }$, which for $3 / 8$ inch $(10 \mathrm{~mm})$ maximum size aggregate (MSA) is $0.02 \mathrm{Nmm} / \mathrm{mm}^{2}$, and $f_{\text {cmo }}$ is equal to 10 $\mathrm{MPa}^{8} . f_{c m}$ is the average 28-day compressive strength. The strength data that is available is the strength after more than 2 years (840 days). The 28-day compressive strength of the concrete specimen was 6,100 psi (42.14 MPa). By substituting in equation 7 ,

$G_{f}=\left(0.02 \times 10^{3} \mathrm{Nm}^{-1}\right)\left(\frac{42.14 \mathrm{MPa}}{10 \mathrm{MPa}}\right)^{0.7}=54.7 \mathrm{Nm}^{-1}$

Irwin $^{9}$ showed that the energy release rate and the stress intensity factor approaches are equivalent. For linear elastic behavior, considering only mode I and plane stress condition:

$$
G_{I}=\frac{K_{I}^{2}}{E}
$$

The measured elastic modulus for the concrete specimen was $E=2.625 \times 10^{6}$ psi $(18.1$ $\mathrm{GPa})$. Substituting in Equation 8:
$K_{I}=\sqrt{G_{f} \cdot E}=\sqrt{\left(54.7 \mathrm{Nm}^{-1}\right)\left(18.1 \times 10^{9} \mathrm{~Pa}\right)} \approx 1 \mathrm{MPa} \sqrt{\mathrm{m}}$

A Poisson's ratio, $v$, value of 0.2 , and a coefficient of friction, $\mu$, value of 0.35 are assumed for the concrete specimen. The representative parameter values for the concrete specimen are shown in Table 2:

Table 2 Concrete specimen Material properties

\begin{tabular}{|c|c|}
\hline Crack Orientation & Random \\
\hline Crack Location & Random \\
\hline Crack Length, $2 a$ & $0.4-1.5 \mathrm{~mm}$ \\
\hline Poisson's Ratio, $v$ & 0.2 \\
\hline Young's Modulus, $E$ & $18.2 \mathrm{GPa}$ \\
\hline Fracture Toughness, $K_{I C}$ & $1 \mathrm{MPa} \sqrt{\mathrm{m}}$ \\
\hline Crack Density, $\Gamma$ & 0.0444 \\
\hline Friction Coefficient, $\mu$ & 0.35 \\
\hline Crack Initial Angle, $\theta$ & $4.9^{\circ}$ \\
\hline
\end{tabular}

All representative parameter values for concrete were taken from Table 2 except the crack lengths which were taken from Table 1. Figure 6 shows the concrete specimen's cross section with the boundary dimensions for the input algorithm.

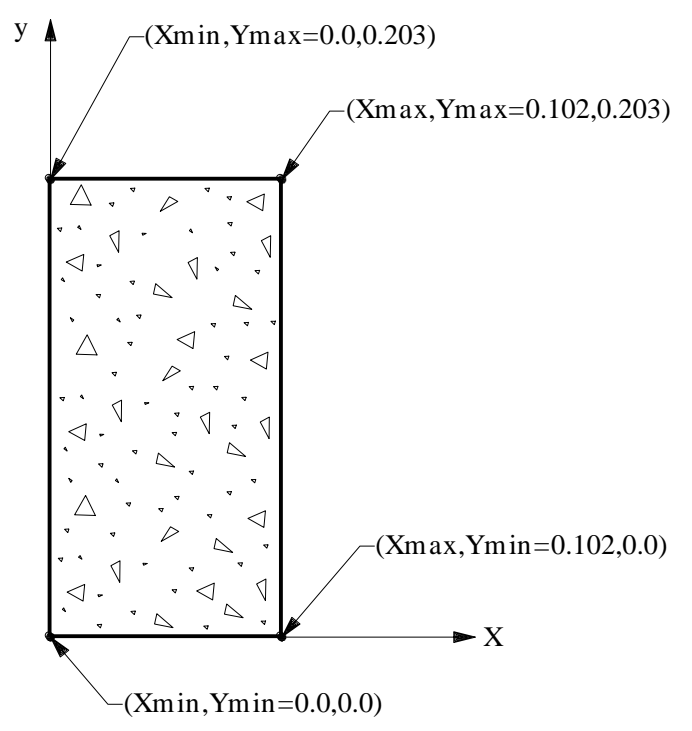

Figure 6 Concrete specimens boundary input in algorithm

The algorithm above generates the crack pattern, which represents the pre-load cracking status. As mentioned earlier, the Du model was 
developed by making a series of modifications to the code of the displacement discontinuity method of Crouch and Starfield ${ }^{11}$. The computer program is called MCPP (Multiple Crack Propagation Program). It is a two dimensional boundary element code which simulates the multiple crack growth, interaction, and coalescence in materials. The input file is prepared from the rectangular boundary of the concrete specimen's cross section, shown in Figure 7, and its information is kept in a file named Bouninput.dat1.

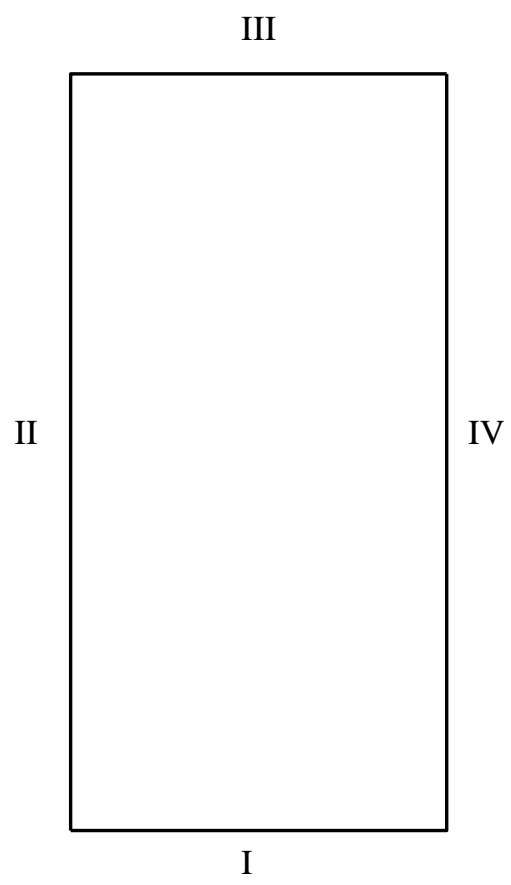

Figure 7 Rectangular boundary

The parameters which are read into the program from Bouninput.dat1 are:

Numbs, Xlow, Ylow, Xlow, Yhigh, An low, An high, Kode, BVS, BVN, Al low, Al.high.

Where:

Numbs $=$ number of straight line boundary segment used to define boundary contours.

(Xlow, Ylow) and (Xhigh, Yhigh) are the beginning and ending coordinates of boundaries I, II, III, IV.

Kode $=1$ indicates that $\sigma_{s}$ and $\sigma_{n}$ are prescribed.

$B V S=\quad$ resultant shear strength $\left(\sigma_{s}\right)$ or shear displacement $\left(u_{s}\right)$
$B V N=$ result ant normal-strength $\left(\sigma_{n}\right)$ or normal displacement $\left(u_{n}\right)$

An low $=$ minimum angle $=0^{\circ}$

An High $=$ maximum angle $=180^{\circ}$

Al low $=$ minimum crack length

Al High = maximum crack length

\section{DISCUSSION}

Using the random crack distribution shown in Figure 5, and employing the Du model, with the axial displacement of 0.00001 meters for 50 iteration, will produce the image shown in Figure 8 . The material properties used are tabulated in Table 2. The material in the model was subjected to an axial strain of $0.25 \%$.

$\left(\varepsilon_{a}=\frac{(0.00001 \text { meter })(50 \text { iterations })}{0.203 \text { meter }}=0.0025=0.25 \%\right.$ strain $)$

The crack density in Figure 8 was measured to be 0.2173 .

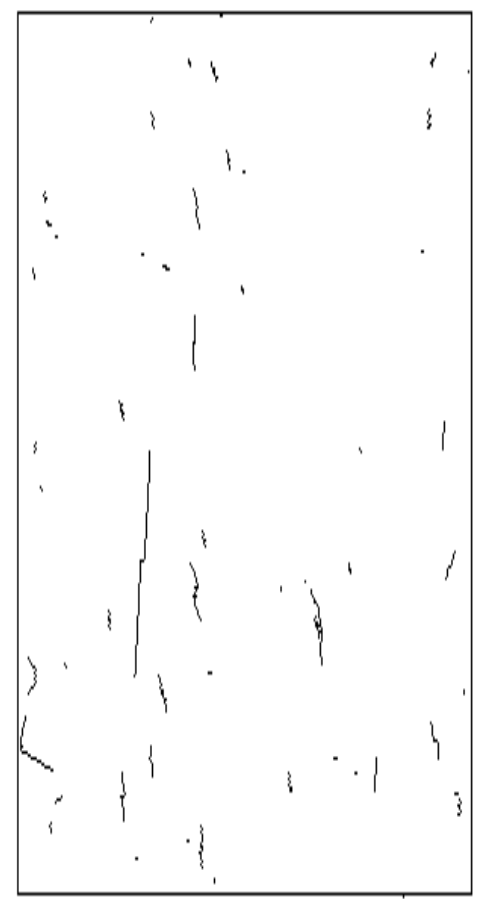

Figure 8 Crack propagation simulation for uniaxial loading

The same no-load model in Figure 5 was then subjected to a confined test, with the same number of iteration and axial strain. The result is shown in Figure 9. It is evident that the 
number of cracks propagating from the original cracks, and also the number of post-load generated cracks was reduced. The resulting crack density was measured to be 0.1878 . There was about $15 \%$ reduction in crack density due to presence of confining stress.

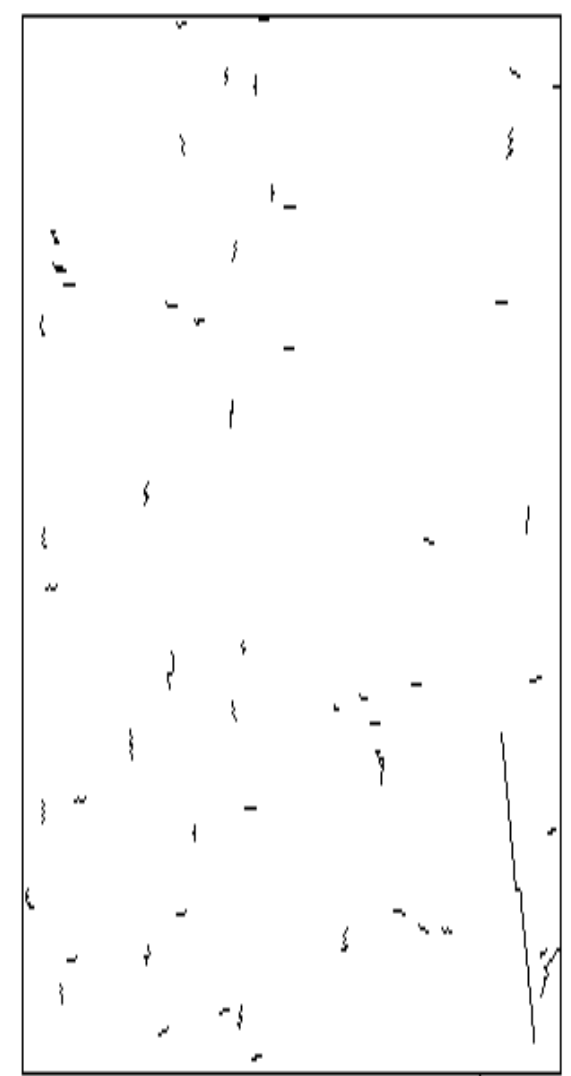

Figure 9 Crack propagation simulation for fully confined condition

The Du model results are consistent with the experimental results. The experimental results showed that the crack density reduced when confining stress was used ${ }^{1}$. The Du model also showed the same behavior. Experimental measurements indicated that the average crack length distribution strongly depends on the confining stress. The results obtained from the Du model, also indicates that the average crack length decreased when confinement was used. Most crack in the Du model have an orientation subparallel to the direction of maximum applied stresses. The definite crack orientation in the Du model is due to the fact that there are no aggregates in the model and it is similar to testing a mortar specimen. The application of the $\mathrm{Du}$ model to concrete crack growth simulation should be enhanced to include hard inclusions, i.e. aggregates, in the model.

\section{CONCLUSIONS}

The crack growth simulation model used the initial pre-load crack pattern, and the elastic constants of the concrete specimens, to generate crack growth with increasing compressive loads. The results from uniaxial and confined specimens were compared against the results obtained from the crack growth model. The differences in crack densities obtained from the crack growth model for the uniaxial and confined loading conditions were identical to the results obtained from the experiments.

The crack simulation growth model does not take aggregates into account. A viable model for crack growth in concrete should incorporate aggregates into the simulation. This could be achieved by randomly distributing pockets of a higher elastic modulus elements within the modeled matrix to simulate the aggregates in concrete.

\section{REFERENCES}

[1] Nemati, K.M., Monteiro, P.J.M., and Scrivener, K.L. "Analysis of Compressive Stress-Induced Cracks in Concrete," American Concrete Institute Materials Journal, Volume 95, Number 5, pp. 617-631, September-October 1998.

[2] Yadev, G.D., Dullien, F. A. L., Chatzis, I., and Macdonald, I. F. (1984). "Microscopic Distribution of Wetting and Non-Wetting phases in Sandstone During Immiscible Displacements," Paper SPE 13212, presented at the 1984 SPE Annual Technical Conference and Exhibition, Dallas, Texas.

[3] Zheng, Z. (1989). "Compressive stressinduced microcracks in rocks and applications to seismic anisot ropy and borehole stability," PhD Thesis, Department of Material Science and Mineral Engineering, University of California, Berkeley. 
[4] Nemati, K.M., Monteiro, P.J.M., and Cook, N.G.W. "A New Method for Studying Stress-Induced Microcracks in Concrete," American Society of Civil Engineers, Journal of Materials in Civil Engineering, Volume 10, Number 3, pp. 128-134, August, 1998.

[5] Nemati, K.M. and Monteiro, P.J.M. "A New Method to Observe ThreeDimensional Fractures in Concrete Using Liquid Metal Porosimetry Technique," Cement and Concrete Research, Volume 27, Number 9, pp. 1333-1341, September 1997.

[6] Moran B. and Sih, C. F. (1987). "A General Treatment of Crack Tip Contour Integrals," International Journal of Fracture , 35, 295-310.

[7] Irwin, G. R. (1956). “Onset of Fast Crack Propagation in High Strength Steel and Aluminium Alloys," Sagamore Research Conference Proceedings , 2, 289-305.

[8] Mehta, P. K. and Monteiro, P. J. M. (2014). "Concrete- Structure, Properties, and Material," McGraw Hill, Forth Edition.

[9] Irwin, G. R. (1957). "Analysis of Stresses and Strains near the End of a Crack Transversing a Plate," Trans ASME, Journal of Applied Mechanics , 24, 361364.

[10] Du, W. (1994). "Numerical modeling of mixed mode multiple crack propagation and its application to the simulation of non-linear rock deformation and borehole breakout," Ph.D. Thesis, University of Arizona.

[11] Crouch, S. L. and Starfield, A. M. (1983). "Boundary Element Method in Solid Mechanics," George Allen \& Unwin, London.

[12] Kemeney, J. M. (1991). “A Model for Non-linear Rock Deformation Under Compression Due to Sub-critical Crack Growth," International Journal of Rock Mechanics and Mining Science and
Geomechanics Abstracts, 28(6), 459467. 